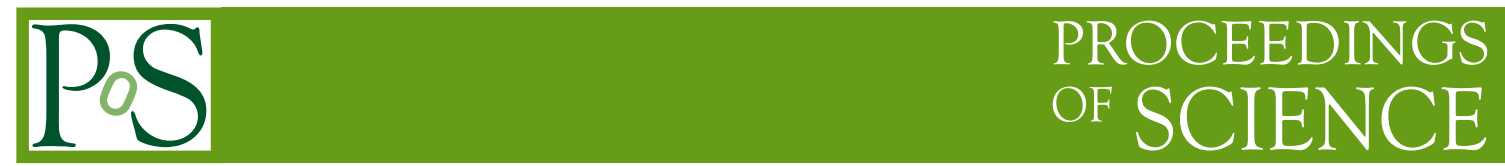

\title{
Progress in Beyond the Standard Model Theories
}

\author{
James D. Wells* \\ CERN, Theory Group, CH-1211 Geneva 23, Switzerland \\ E-mail: james.wells@cern.ch
}

I present a review of developments in beyond the Standard Model physics. The emphasis here is on how experimental progress of the last few years has affected our thinking on theories.

35th International Conference of High Energy Physics - ICHEP2010,

July 22-28, 2010

Paris France

${ }^{*}$ Speaker. 
My task at this conference is to summarize the recent advances in beyond the Standard Model (SM) physics. The writing that follows is intended to be short essay style of various thoughts I delivered in the talk. Plots, more equations, more references, nice pictures and complementary text are provided in the actual talk [1]. Comprehensiveness is out of the question for a short presentation like this. Instead I shall present what I think are some of the most interesting themes that have been seizing the community in the last year or two, if not for much further in the past, with emphasis on connection to experimental results. A noticeable exception to this is the astrophysical anomalies (DAMA/Libra, Pamela, CoGent, etc.) that are to be summarized elsewhere.

There are three themes that stand out to me. The first theme springs from the recognition that the LHC is the most powerful street lamp in history. We make fun of the drunk man who looks for his keys under the lamppost because it is the only place he can see; nevertheless, it is not an altogether unreasonable strategy. Some of our ideas in beyond the SM physics are not pretty, and may not even be very motivated by any outstanding problem that we have facing us today, but they can be seen at the LHC.

Lamppost physics is generally a waste of time unless it leads experimentalists to analyses that can find new physics that would not be noticed otherwise. Some of the ideas presented at this meeting - hidden sector, exotic spin particles, quirks, many exotic Higgses, etc. - have this quality. By taking them seriously we increase the prospects of finding new physics - albeit ever so slightly - and it increases the interpretative power of the data.

In my mind the most interesting lamppost physics topics are ones that involve the Higgs boson. That is for several reasons. First, we really do not have an idea how electroweak symmetry breaking and elementary particle mass generation operates. Higgs boson physics is mysterious, and the best way to humbly acknowledge this fact is be very broad in our approach to its phenomenology. The second reason to look under the lamppost in the area of Higgs physics is the accidental narrowness of its width in the precision electroweak best fit interval $(115 \mathrm{GeV}$ to about $170 \mathrm{GeV})$. Over much of the lower range the most copious decay is to $b$ quarks, which couples very weakly $y_{b}=m_{b} / v$ and thus provides no substantial partial width. This in turn makes any new physics decay capable of competing with and even overwhelming the SM decay modes. There have been many papers over the last few years exploiting exactly this kind of characteristic of the Higgs sector to identify new, exotic signals possible at the LHC. I do not intend to talk about this much. There are countless examples, including one given at this conference (see for example Nandi's talk [2]).

The second theme that has stood out over the last few years, which is an ever-present activity in the history of forefront science, is the pursuit of explanations for anomalies that need to be explained. We have plenty of anomalous in our field. These include the Pamela positron "excess", DAMA/Libra seasonal variation, $g-2, B_{s}$ dimuon $\mathrm{CP}$ asymmetry, the difference between neutrino and antineutrino properties, forward-backward asymmetry of the top quark, etc. As with most anomalies they are likely to "go away" with time. Nevertheless, all new physics had to start as a anomaly before a discovery, so it is a valid motivation to consider new physics that could explain it.

A good recent example in this category is the $t \bar{t}$ forward-backward asymmetry. Since QCD is a $\mathrm{C}, \mathrm{CP}$ and $\mathrm{P}$ invariant theory, it is often surprising to people to hear that it can give rise to a charge asymmetry, or forward-backward asymmetry. We are used to forward-backward asymmetries in $Z$ physics, but there it is obvious and at tree-level by virtue of the $\gamma_{5}$ interaction of the $Z$ boson with 
the fermions. However, for QCD the gluons do not interact with quarks via $\gamma_{5}$. Yet, at order $\alpha_{s}^{3}$ the asymmetry arises, due to interference of tree-level diagram with a box loop diagram. The full amplitude at this one-loop order is invariant under reversing the arrows of all the fermions, but it is not invariant under reversing the arrows of the top quark. And that is all that is needed to produce the asymmetry. An analogous effect in QED tells us that there is an $\alpha^{3}$ forward-backward charge asymmetry with the muon in $e^{+} e^{-} \rightarrow \mu^{+} \mu^{-}$.

The theory prediction at Tevatron is $A_{F B}^{t h}=5 \pm 1 \%$ (see, for example [3]). The experimental predictions have been consistently high, starting from Schwarz's thesis value of $0.20 \pm 0.11^{\text {stat }} \pm$ $0.047^{\text {stat }}$ to the value reported last year of $0.193 \pm 0.065^{\text {stat }} \pm 0.024^{\text {syst }}$ with $3.2 \mathrm{fb}^{-1}$ of CDF data [7]. Eppig reported [8] at ICHEP the latest CDF result of $0.150 \pm 0.050 \pm 0.024$ with $5.3 \mathrm{fb}^{-1}$. D0 reported a result at ICHEP, but it is uncorrected and I do not understand what it means.

If we would like to obtain a large effect at tree-level for the forward-backward asymmetry, the most effective way to do it is to introduce a $\gamma_{5}$ in the $t \bar{t}$ production cross-section. The simplest way to do that is through the so-called $S U(3)_{L} \times S U(3)_{R}$ chiral color theories. When this breaks to $S U(3)_{c}$ there are 8 massless gluons that couple vectorially to the quarks, and 8 massive axigluons that couple axial vectorially to the quarks. The axigluon mediation introduces maximal charge asymmetry effects as the tree-level $\bar{t} \gamma^{\mu} \gamma^{5} t$ is relative $C$ odd to $\bar{t} \gamma^{\mu} t$. The trouble with this approach is that the asymmetry induced goes the wrong way. It introduces a large negative contribution, whereas we are looking for a positive contribution. The effect is so pronounced that the limits on axigluons from $A_{F B}^{t}$ may be stronger than from direct bump hunting searches. At the moment, limits from both are in the neighborhood of about a $\mathrm{TeV}$ [9].

One can try more general $g_{V}$ vs. $g_{A}$ couplings to see if there is a way to get a large asymmetry without running into conflict with other eperiments. Ferrario and Rodrigo [4] have done a good job of exploring this parameter space. However, it turns out that whenever one gets the asymmetry to be enhanced in the positive direction, one runs into trouble with the $t \bar{t}$ invariant mass distributions. Thus, the axigluon approach does not work [6].

This does not exhaust all the possibilities for these theories. There are many more that can give rise to the asymmetry without causing too much trouble for other observables, including an axigluon theory that has opposite sign axial vector couplings for the light quarks compared to the top quark. Other ideas include $t$-channel $Z^{\prime}$ with $Z-u-t$ coupling, warped RS KK gluons, and many other ideas.

The third theme of BSM physics to touch upon is the push for theories to be complete and natural. A goal of particle physics is to understand everything, and this includes constructing "natural theories" that explain dark matter, baryogenesis, the hierarchy of the Planck scale to the weak scale, etc. We are not content with a different theory explaining a different puzzle - we strive for a complete theory that answers all of our questions.

Supersymmetry is perhaps the leading candidate theory to understand everything at or near the weak scale. However, one of the challenges is to understand how the lightest Higgs boson of supersymmetry can evade the $115 \mathrm{GeV}$ mass limit from direct searches at LEP. (See also Csaki's talk [10] on this subject, but from the different perspective of enabling Higgs masses below 115 $\mathrm{GeV}$ to evade discovery.) The lightest Higgs boson mass is limited at leading order to be less than $m_{Z}$. With radiative corrections it can be shifted upwards via $\delta m_{h}^{2} \propto \ln \tilde{m}_{t} / m_{t}$. For $m_{h}>115 \mathrm{GeV}$ this requires $\tilde{m}_{t}>1 \mathrm{TeV}$. Some view this large of a value for $\tilde{m}_{t}$ to constitute an unacceptably large 
finetuning in the electroweak sector of the supersymmetric theory. I am not sure I agree to that conclusion, but it is held widely so I will proceed with the discussion.

One of the simplest cures is to go beyond the MSSM and include a singlet Higgs in the superpotential $\Delta W=\lambda S H_{u} H_{d}+\cdots$. The $F$-term contribution to the potential yields a term of the size $\delta m_{h}^{2}=\lambda^{2} v^{2} \sin ^{2} 2 \beta$ where $\beta$ is the same angle used to define $\tan \beta=v_{u} / v_{b}$. This appears to solve all the problems immediately, as $\lambda$ is an a priori arbitrary coupling and therefore the Higgs boson mass can be as large as we wish.

The trouble though with that approach is that for rather small values of $\lambda$, it will blow up at low energies from renormalization group scaling. One of the major interests in supersymmetry is its apparent unification of couplings at $\sim 10^{16} \mathrm{GeV}$. This would be greatly compromised if it turned out that $\lambda$ blew up well before then, because $\lambda$ effects the Yukawa couplings and gauge couplings through higher order affects and would disrupt the reliability of their quantum rescalings as well. The unification of couplings would then be merely an accident. Lodone's ICHEP talk, for example, discusses this issue [11], and concludes that the further advantages of this approach - namely, the amelioration of the flavor problem by using much heavier 1st and 2nd generation squarks - can outweigh the negative of losing perturbative gauge coupling unification. It is of course up to nature to decide if such a traumatic trade-off is worth it. Personally, I am more in favor of a split supersymmetry kind of approach [12] to the Higgs mass problem than to having the theory go non-perturbative so quickly, but admittedly I romanticize gauge coupling unification more than most.

Finally, let us remark that experimental progress has played a decisive role in the rise or demise of various beyond the SM theories of late. The Higgs boson mass constraints, precision electroweak analysis, and direct searches for new particles have been devastating to many ideas, but mildly supportive to others. There is continual effort to explain more. Our questions and ideas are more refined with more knowledge and insight. There is much anticipation for further results from all experiments, including at the high-energy frontiers of the Tevatron and LHC.

\section{References}

[1] J.D. Wells, "Progress in Beyond the Standard Model Theory", ICHEP 2010 (27 July 2010) http://indico.cern.ch/contributionDisplay.py?contribId=99\&confId=73513

[2] S. Nandi, "New mechanism for neutrino mass generation", ICHEP 2010 (24 July 2010) http://indico.cern.ch/contributionDisplay.py?contribId=139\&sessionId=55\&confId=73513

[3] J. H. Kuhn, G. Rodrigo, “Charge asymmetry of heavy quarks at hadron colliders," Phys. Rev. D59, 054017 (1999). [hep-ph/9807420]; O. Antunano, J. H. Kuhn, G. Rodrigo, “Top quarks, axigluons and charge asymmetries at hadron colliders," Phys. Rev. D77, 014003 (2008). [arXiv:0709.1652 [hep-ph]].

[4] P. Ferrario, G. Rodrigo, "Massive color-octet bosons and the charge asymmetries of top quarks at hadron colliders,” Phys. Rev. D78, 094018 (2008). [arXiv:0809.3354 [hep-ph]].

[5] S. Jung et al., Phys. Rev. D81, 015004 (2010). [arXiv:0907.4112 [hep-ph]].

[6] R. S. Chivukula, E. H. Simmons, C. -P. Yuan, "Axigluons cannot explain the observed top quark forward-backward asymmetry," [arXiv:1007.0260 [hep-ph]].

[7] G. Strycker et al., "Measurement of the Forward-Backward Asymmetry in Top Pair Production in $3.2 \mathrm{fb}^{-1}$ of $p \bar{p}$ collisions at $\sqrt{s}=1.96 \mathrm{TeV}$, " CDF Note 9724 (March 17, 2009). 
[8] A. Eppig, "Studies of Top Quark Properties at CDF," ICHEP 2010 (23 July 2010) http://indico.cern.ch/contributionDisplay.py?contribId=1134\&sessionId=48\& confId=73513

[9] K. Nakamura et al. (Particle Data Group), J. Phys. G37, 075021 (2010).

[10] C. Csaki, "A critical overview of electroweak symmetry breaking," ICHEP 2010 (26 July 2010) $\mathrm{http} / / /$ indico.cern.ch/contributionDisplay.py?contribId=84\&sessionId=13\&confId=73513

[11] R. Barbieri et al., JHEP 1008, 024 (2010). [arXiv:1004.2256 [hep-ph]].

[12] See, for example, J.D. Wells, "Implications of supersymmetry breaking with a little hierarchy between gauginos and scalars," hep-ph/0306127; N. Arkani-Hamed, S. Dimopoulos,

"Supersymmetric unification without low energy supersymmetry and signatures for fine-tuning at the LHC," JHEP 0506, 073 (2005). [hep-th/0405159]; G. F. Giudice, A. Romanino, "Split supersymmetry," Nucl. Phys. B699, 65-89 (2004). [hep-ph/0406088]. J. D. Wells, "PeV-scale supersymmetry," Phys. Rev. D71, 015013 (2005). [hep-ph/0411041]. 